\title{
Hyaline membrane disease in relation to fibrinolytic activity
}

\author{
L. SINCLAIR
}

From Westminster Children's Hospital, London

There are several points of interest about hyaline membrane disease in relation to any discussion of fibrinolytic activity. In paediatrics hyaline membrane disease is a persisting cause of mortality. Between $40 \%$ and $60 \%$ of premature infants dying in the first five days of life are said to die of this condition. The diagnosis falls to the pathologist who examines the lungs after death, for during life the patient will have been said to have had the 'respiratory distress syndrome'. Although many theories have been put forward to explain why apparently 'hyaline' material is laid down on the alveolar epithelium, only certain aetiological factors are known to be common to most patients. It is most common in premature infants, particularly those of mothers who are diabetic or have the so-called 'pre-diabetic' state, and in infants born by Caesarian section, usually after prolonged labour. The deposition of pinkstaining hyaline material in the alveoli, as seen in sections stained with haematoxylin and eosin, also occurs in other pathological conditions, and is found in the lungs of patients dying of such widely differing conditions as cardiac failure, pneumonia, and after the inhalation of irritating gases.

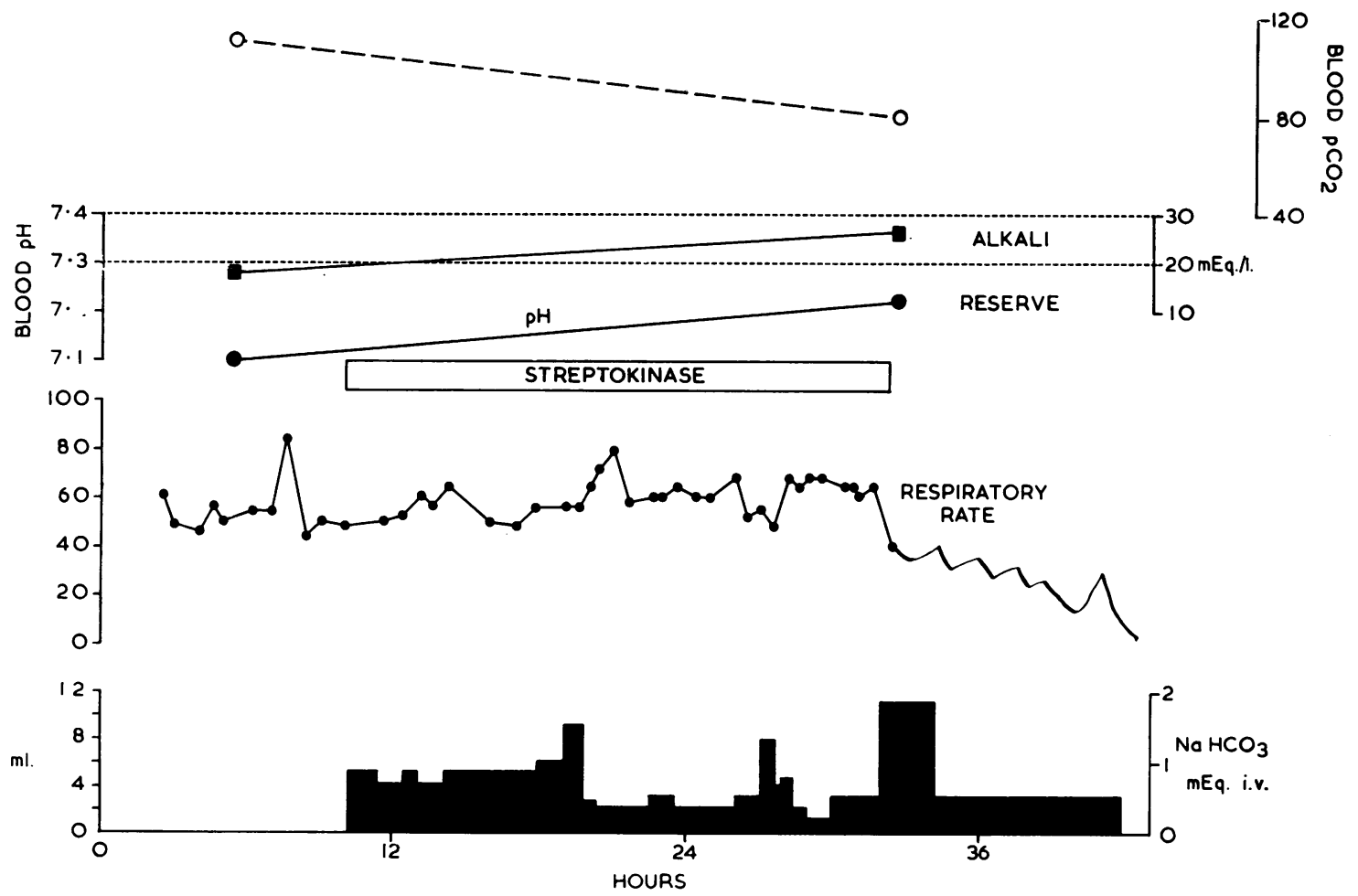

FIG. 1. Chart of response of infant with hyaline membrane disease to therapy with streptokinase and intravenous sodium bicarbonate and glucose (inhalation at the rate of 100 units per hour). 
More light on the nature of this membrane has recently been shed by Gitlin and Craig (1956) who used the immunofluorescence of anti-fibrin antibodies to identify the basic matrix of the 'membrane' as fibrin, and this work has been supported by Van Breemen, Neustein, and Bruns (1957). It is presumed that the pathogenesis is as follows. The initial insult produces pulmonary oedema fluid which contains protein and mucoprotein. The fluid is absorbed either efficiently or not so, leaving a deposit of fibrin and other substances behind. It was originally claimed by Lieberman (1959) that the lungs of infants dying of hyaline membrane disease had a low concentration of activator as $80 \%$ of control lungs had a normal concentration of activator, and Lieberman suggested than an inhibitor had been released in high concentration from a damaged placenta.

In the light of these observations it was decided to treat two infants born at the Westminster Hospital. Each was treated by giving inhalations of streptokinase in aerosol through a face mask. Therapy was also maintained with intravenous sodium bicarbonate and glucose solution as described by Usher (1961) and Warley and Gairdner (1962). The first infant recovered after 24 hours' therapy with streptokinase aerosol and three days' therapy with buffer and glucose solutions intravenously. The second did not survive. She was born by Caesarian section prematurely and respiration was difficult to establish after delivery. The response to therapy is shown in Fig. 1, from which it will be seen that the severe respiratory acidosis and carbon dioxide retention was improved after 24 hours' treatment, but the infant developed respiratory difficulty. At necropsy the lungs showed the typical changes of hyaline membrane disease although the membrane had shown evidence of dissolution, a finding usually made during the recovery phase, but the membrane had persisted nevertheless and therapy with this agent must be considered unsatisfactory.

More light on the problem has been shed recently by Ambrus, Weintraub, Dunphy, Dowd, Pickren, Niswander, and Ambrus (1963), who showed that plasminogen was absent from both the plasma of normal premature infants and of those with the respiratory distress syndrome. Plasminogen con centration was highest in normal newborn infants and activator was high in the blood of infants wit ${ }_{3}$ the respiratory distress syndrome. It appears likel that activator was liberated from tissue during the course of respiratory distress by anoxia, but in the absence of plasminogen this would not be of significance. Ambrus et al. treated 33 infants with the respiratory distress syndrome in a randomized double-blind investigation. Eleven were treate $\vec{\phi}$ with a placebo and five survived. Eight were treated with streptokinase-activated human plasmin anf only two survived, and 14 were treated with uro응 kinase-activated plasmin and 12 survived. Although the latter results appear encouraging, much remains to be uncovered in the study of hyaline membrane disease. It is obvious that a study of plasmin activit店 in arterial and venous blood of infants with this disorder may be another useful tool in the diagnosis and control of treatment by fibrinolytic agents.

I am grateful to Dr. Ian M. Anderson for permission to publish details of his cases, to Dr. H. M. T. Coles fof encouragement and advice, and to Dr. H. D. Brooks for the chemical investigations.

\section{REFERENCES}

Ambrus, C. M., Weintraub, D. H., Dunphy, D., Dowd, J. E., Pickren J. W., Niswander, K. R., and Ambrus, J. L. (1963). Pediatrics 32, 10.

Gitlin, D., and Craig, J. M. (1956). Ibid., 17, 64

Lieberman, J. (1959). New Engi. J. Med., 260, 619.

Usher, R. H. (1961). In CIBA Foundation Symposium on Somatic Stability in the Newborn, edited by G. E. W. Wolstenholme and? M. O'Connor, p. 92. Churchill, London.

Van Breemen, V. L., Neustein, H. B., and Bruns, P. D. (1957): Amer. J. Path., 33, 769.

Warley, M. A., and Gairdner, D. (1962). Arch. Dis. Childh., 37, $455 \frac{0}{\circ}$

DR. CROSBIE asked if it would not be more logical to pus the infant in a high-pressure oxygen chamber and give ith plasminogen, because there would appear to be activator? available but not plasminogen. The greatly increased oxygen pressure would allow oxygen to pass through the circulation by diffusion while fibrinolysis was going on. $>$ DR. SINCLAIR replied that high concentrations of oxygen were dangerous to infants because of the risk of producing retrolental fibroplasia.

DR. MCNICOL felt that hyaline membrane disease was anN ideal situation for the use of a concentrated preparation of plasmin administered by aerosol. 